\title{
Phase II/III Trial
}

National Cancer Institute

\section{Source}

National Cancer Institute. Phase II/III Trial. NCI Thesaurus. Code C15694.

A type of clinical study that combines elements characteristic of traditional Phase II and Phase III trials. 